OPEN ACCESS

Edited by:

Carmen Criscitiello,

European Institute of Oncology

(IEO), Italy

Reviewed by:

Paolo Tarantino,

European Institute of Oncology

(IEO), Italy

Julio César de la Torre-Montero,

Comillas Pontifical University, Spain

${ }^{*}$ Correspondence:

Qiu Li

liqiu@scu.edu.cn

Specialty section:

This article was submitted to

Women's Cancer,

a section of the journal

Frontiers in Oncology

Received: 26 April 2020

Accepted: 26 June 2020

Published: 04 August 2020

Citation:

Wu Q, Liao W, Zhang M, Huang J,

Zhang P and Li Q (2020)

Cost-Effectiveness of Tucatinib in

Human Epidermal Growth Factor

Receptor 2-Positive Metastatic Breast

Cancer From the US and Chinese

Perspectives. Front. Oncol. 10:1336.

doi: 10.3389/fonc.2020.01336

\section{Cost-Effectiveness of Tucatinib in Human Epidermal Growth Factor Receptor 2-Positive Metastatic Breast Cancer From the US and Chinese Perspectives}

\author{
Qiuji Wu ${ }^{1,2}$, Weiting Liao ${ }^{1,2}$, Mengxi Zhang ${ }^{1,2}$, Jiaxing Huang ${ }^{1,2}$, Pengfei Zhang ${ }^{1,2}$ and \\ Qiu $L i^{1,2 *}$
}

${ }^{1}$ Department of Medical Oncology, Cancer Center, West China Hospital, Sichuan University, Chengdu, China, ${ }^{2}$ West China Biomedical Big Data Center, Sichuan University, Chengdu, China

Background: The clinical evaluation of HER2CLIMB trial showed a 21. 9-month median overall survival with the triplet regimens of tucatinib, capecitabine, and trastuzumab (TXT) for patients with human epidermal growth factor receptor 2 (HER2) -overexpressing metastatic breast cancer. From the payer's perspective of the United States and China, a cost-effectiveness analysis was conducted to evaluate the costs and benefits of adding tucatinib in this study.

Methods: We constructed a Markov model for the economic evaluation of adding tucatinib to trastuzumab plus capecitabine in patients with HER-2 positive metastatic breast cancer in the United States and China. The model was conducted with a 10-year time horizon, and the health status was divided into three states: progression-free survival, progressing disease, and death. The health utility scores were consistent with published literature with similar patient status. The transition probabilities were derived from the survival data of the HER2CLIMB study. The unit prices of medicines were obtained from the West China Hospital, Red Book, and published literature. Outcomes were measured in quality-adjusted life-years (QALYS), and incremental cost-effectiveness ratio, which robustness was evaluated by deterministic and probabilistic sensitivity analyses.

Results: Compared with the two-drug regimen of trastuzumab plus capecitabine (TX), the addition of tucatinib increased 0.21 QALY, with an increasing cost of $\$ 146,995.05$ and $\$ 19,022.97$ in the United States and China, respectively. The incremental costeffectiveness ratios (ICERs) for the TXT versus TX was $\$ 699,976.43$ in the U.S. and $\$ 90,585.57$ in China, both of which are higher than their respective threshold of willingness to play. Deterministic sensitivity analysis shows that the price of tucatinib is the parameter that has the most significant impact on ICERs, but it does not change the results of the model. Probability sensitivity analysis shows that the probability of cost-effective for TXT is 0 in the base case. 
Conclusion: In the United States and China, tucatinib combined with trastuzumab and capecitabine is not cost-effective for patients with HER-2 positive metastatic breast cancer.

Keywords: tucatinib, metastatic breast cancer, HER2-positive, cost-effectiveness, Markov model

\section{INTRODUCTION}

Cancer statistics in 2020 show that the incidence of breast cancer ranks first among female tumors (276,480 cases) and the secondhighest mortality rate $(42,170$ death) in the United States (1). In China, breast cancer is the most common cancer among women, with 268.6 thousand people diagnosed with breast cancer in 2015 (2). These cases in China account for $17.6 \%$ of all newly diagnosed breast cancers and $11.1 \%$ of all breast cancer deaths worldwide $(2,3)$. An estimated $5.7 \%$ of incidence cases of breast cancer cases are distributed in Stage IV or metastatic in the United States (4). In China, up to $21.4 \%$ of patients with breast cancer have distant metastases at the initial diagnosis (5). Even after undergoing surgery and standard treatment, $20-30 \%$ of patients will relapse within 10 years, of which two-thirds are distant metastases $(6,7)$.

Human epidermal growth factor receptor 2 (HER2), as an oncogene for tumorigenesis, is overexpressed in $20-25 \%$ of invasive breast cancers, closely related to invasion, metastasis, and prognosis (8-10). In the advent of trastuzumab, anti-HER2 therapies have led to a significant improvement in overall survival in early and advanced patients with HER2-positive breast cancer. However, most patients ultimately develop the progressive disease and die. Furthermore, up to $40-50 \%$ of HER2-positive breast cancer patients will develop brain metastases $(11,12)$. Better options for the prevention and treatment of brain metastases are needed. There is no standard treatment for patients with HER2-positive metastatic breast cancer (MBC) that have progressed after treatment with trastuzumab, pertuzumab, and trastuzumab emtansine (T-DM1). Treatment options at this time include lapatinib in combination with capecitabine, trastuzumab and other chemotherapy (such as vinorelbine or gemcitabine), or participation in a clinical trial. A substantial number of novel anti-HER2 treatments are being investigated extensively in the preclinical and clinical settings, including novel antibody-drug conjugates (such as trastuzumab deruxtecan), other small-molecule tyrosine kinase inhibitors (TKIs) (such as tucatinib, neratinib, and pyrotinib).

Tucatinib is an orally bioavailable, small-molecule tyrosine kinase inhibitor. Different from other small molecules TKIs, tucatinib can selectively inhibit HER2 (13). In June 2017, tucatinib received orphan drug designation from the US Food and Drug Administration (FDA) for treatment of breast cancer patients with brain metastases (14). Tucatinib, trastuzumab, and capecitabine (TXT) were shown to improve overall survival compared with trastuzumab and capecitabine in the HER2CLIMB (ClinicalTrials.gov identifier, NCT02614794.) clinical trial (21.9 vs. 17.4 months), besides also significantly extending progression-free survival (PFS) compared with trastuzumab and capecitabine (TX) (7.8 vs. 5.6 months) (15).
Based on these data, the FDA approved tucatinib in combination with chemotherapy (trastuzumab and capecitabine) for patients with HER2-positive MBC who have received one or more prior treatments in April 2020 (16).

The implications of tucatinib in the treatment of HER2 positive MBC are considerable, given the significant potential population of patients eligible to receive the therapy. Once tucatinib is approved in more countries, the widespread use of the drug may substantially increase the costs of breast cancer care. Moreover, the global breast cancer burden in women is rising in countries regardless of income level. Under the influence of COVID-19, the world economy and health has changed in profound and almost universal ways, adding more pressure to the growing shortage of limited medical resources. Cost-effectiveness analysis as an important tool for assessing whether new therapies provide clinical benefits at a reasonable cost is increasingly recognized. In this study, we assessed the appropriate price range of tucatinib for Chinese and US payers through cost-effectiveness analysis and explored the economics of new drugs in developing and developed countries.

\section{MATERIALS AND METHODS}

\section{Patients and Intervention}

Our model is to simulate the treatment of patients with HER2positive metastatic or recurrent breast cancer in the HER2CLIMB trial. The hypothetical cohort included patients over 18 years of age who have received a treatment course of trastuzumab, pertuzumab, and trastuzumab emtansine (15). Eligible patients were randomly assigned in a 2:1 ratio to treatment with TXT or TX. Patients in the TXT arm were administered $300 \mathrm{mg}$ tucatinib twice daily throughout the treatment period. Besides, patients in both groups received trastuzumab $(8 \mathrm{mg} / \mathrm{kg}$ for initial therapy followed by $6 \mathrm{mg} / \mathrm{kg}$ for maintenance) on day 1 and capecitabine $(1,000 \mathrm{mg} / \mathrm{m} 2)$ twice daily on days $1-14$ repeated every 3 weeks (15). Patients continued to receive the current treatment plan until unacceptable toxicity, disease progression, withdrawal of consent, or study closure (15).

\section{Model Construction}

We constructed a decision analysis Markov model through TreeAge Pro 2011 software (TreeAge, Williamstown, MA) to simulate the process of TXT or TX treatment for HER-2 positive $\mathrm{MBC}$ and to predict the 10-year costs and survival benefits of the two strategic therapies. A Markov decision process (MDP) is a stochastic model to a final decision process. Unlike the deterministic model, the Markov model results will change each time they are solved even though the initial conditions remain unchanged (17). The model consists of three mutually incompatible health states: progression-free survival 


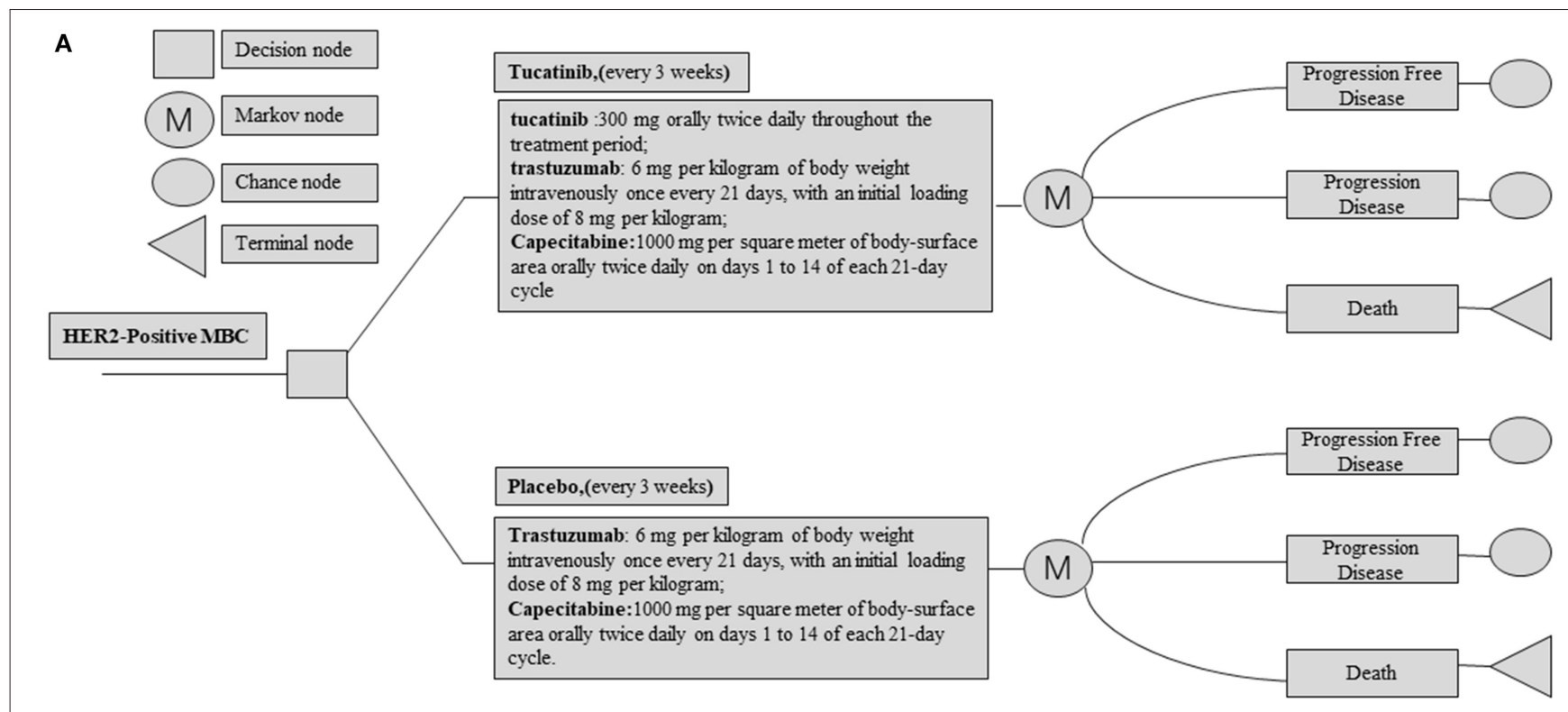

B

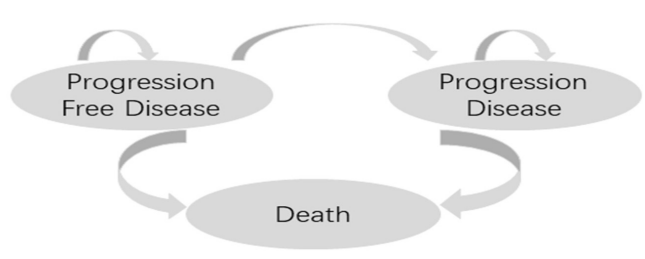

FIGURE 1 | (A) Abbreviated decision tree and Markov model used to compare two strategies for treating patients with HER2-Positive Metastatic Breast Cancer. (B) The influence diagram shows a network of three health states linked by transitional variables.

(PFS), progression disease (PD), and death. The output of this model includes total cost, quality-adjusted life year (QALY), and incremental cost-effectiveness ratio (ICER). ICER is defined as the difference in costs between TXT and TX groups divided by the difference in effects. The ICER between the two groups was compared with the WTP threshold of $\$ 150,000$ per QALY in the USA and $\$ 30,447.09$ /QALY (three times GDP per capita) in China, respectively $(18,19)$. According to the HER2CLIMB trial, all patients in the model were randomly assigned to two groups with PFS status (Figure 1). The cycle length of the model is 3 weeks, which is consistent with the treatment cycle of the patients in the HER2CLIMB trial, and a half-cycle correction was applied (20).

The OS and PFS probabilities were extracted from the published OS and PFS curves of the HER2CLIMB trial by WebPlotDigitizer software (version 4.2; https://apps.automeris. io/wpd/index.zh_CN.html) (15), and these survival data were then used to fit parametric survival models using the algorithm derived by Hoyle et al. (21). The Weibull survival model results of the two groups are shown in Figure 2. Based on the fitted Weibull model, we can estimate the timedependency transition probability from PFS to PD and $\mathrm{PD}$ to death in each cycle using the following formula:
$\left.\mathrm{P}(\mathrm{t} \rightarrow \mathrm{t}+1)=1-\exp \left[\lambda(\mathrm{t})^{\gamma}-\lambda(\mathrm{t}+1)^{\gamma}\right)\right]$, where $\mathrm{t}$ stands for the current cycle number in the Markov model (22). The mortality rate in the PFS state for each age group was estimated based on Chinese and US life tables in the model $(23,24)$.

\section{Cost and Utilities}

The cost of each group is assessed from the perspective of the Chinese and US payers, including the cost of tucatinib, trastuzumab, capecitabine, management of grade 3-4 adverse events (AEs), administration, best supportive care, and test. These costs come from Red Book, published literature, West China Hospital of Sichuan University, and Chinese national drug prices (25-37). The price of lapatinib in China was used in the base-case analysis because the tucatinib is not yet marketed. All expenses are listed in Table 1. We assumed the patients in both groups received best supportive care (BSC) after progression for absent treatment data in the sequence line. US and China costs associated with health care services were inflated to 2020 values according to the US and China consumer price index $(38,39)$. We converted all costs to US dollars [ $\$ 1=¥ 6.9851$ (February 2020)]. A discount rate of $3 \%$ per year was used for the costs and utility value involved in the model (40). The utility value represents the health-related quality of life for each state of health, ranging 


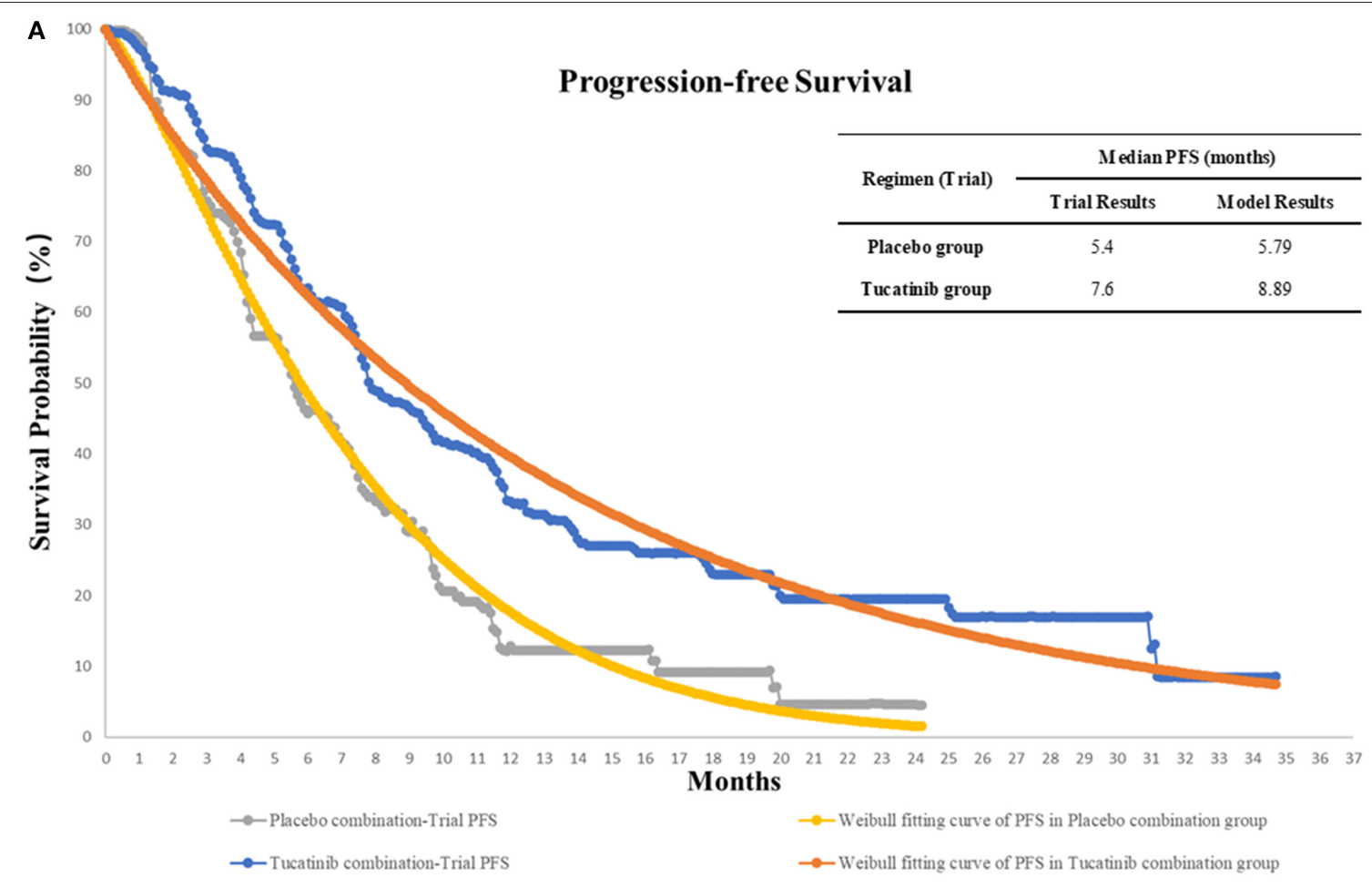

B

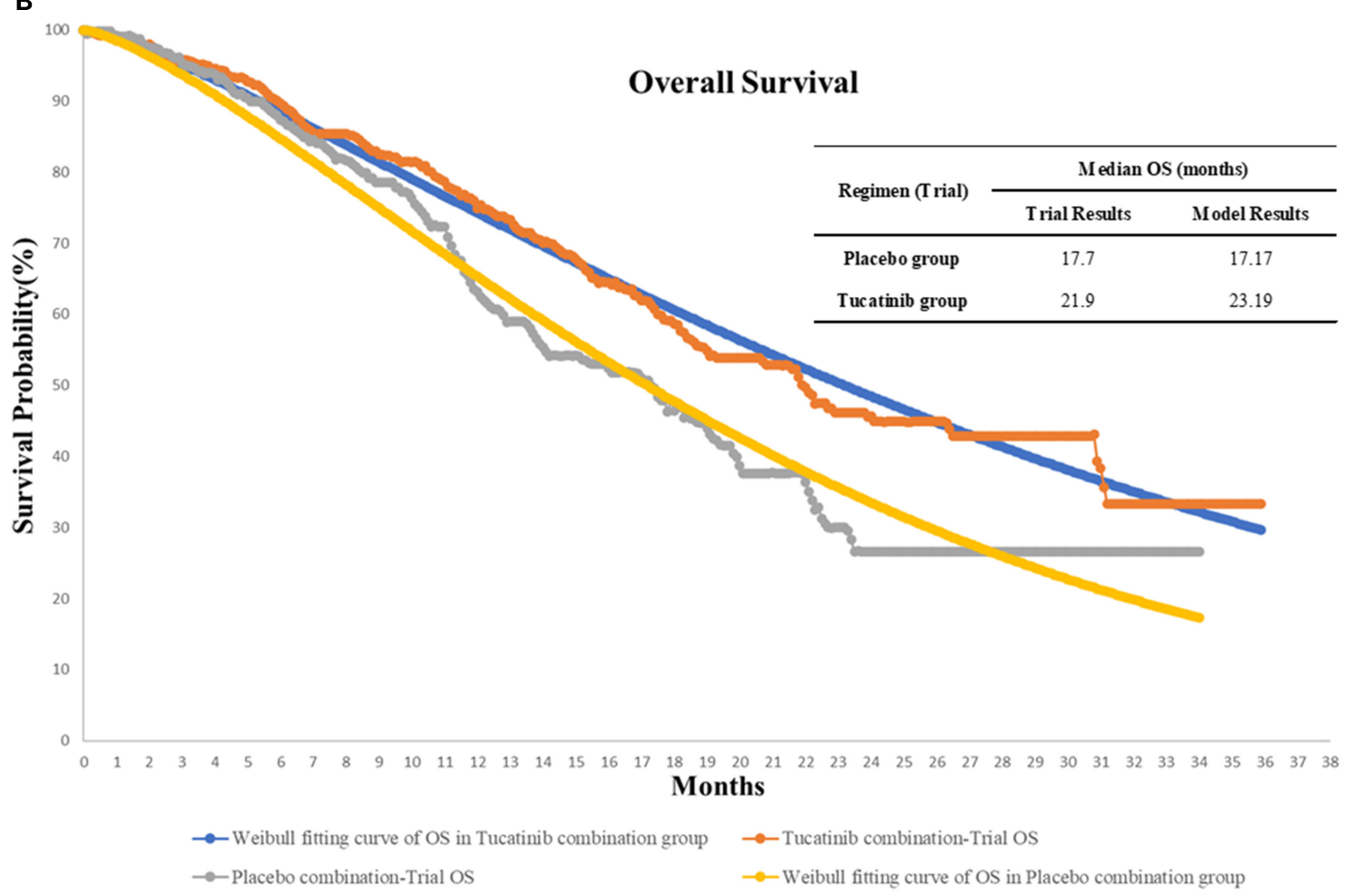

FIGURE 2 | The original Kaplan-Meier PFS (A) and OS (B) curves from the HER2CLIMB trial, Weibull fitting curves, and the validation of our model of treatment strategies for HER2-Positive Metastatic Breast Cancer. OS, overall survival; PFS, progression-free survival. 
TABLE 1 | Model parameters and assumptions.

\begin{tabular}{|c|c|c|c|c|c|}
\hline \multirow[t]{2}{*}{ Parameter } & \multicolumn{2}{|c|}{ USA value } & \multicolumn{2}{|c|}{ China value } & \multirow[t]{2}{*}{ Distribution } \\
\hline & Mean & Range & Mean & Range & \\
\hline Tucatinib per cycle, $\$$ & $12,950(22)$ & $9,065-16,835$ & $1,002.03$ & $701.42-1,302.64$ & $\curlyvee$ \\
\hline Trastuzumab per cycle, $\$$ & $3,669.08(22,23)$ & $2,568.36-4,769.80$ & 952.01 & $666.41-1,237.61$ & Fix in PSA \\
\hline Capecitabine per cycle, $\$$ & $955.50(22,23)$ & $668.85-1,242.15$ & 189.60 & $132.72-246.48$ & Fix in PSA \\
\hline BSC/cycle, \$ & $2,933(24)$ & 2,053.1-3,812.9 & $807(31)$ & $564.9-1,049.1$ & $\curlyvee$ \\
\hline Computed tomography imaging, per cycle, $\$$ & $448(25)$ & $313.6-582.4$ & 84.56 & $59.19-109.9$ & $\curlyvee$ \\
\hline Cost of managing adverse events, per event, $\$$ & & & & & $\curlyvee$ \\
\hline Palmar-plantar erythrodysesthesia & $8.31(26)$ & $5.82-10.80$ & $3.57(26)$ & $2.50-4.64$ & $\curlyvee$ \\
\hline Diarrhea & $1,183.7(26)$ & $828.59-1,538.81$ & $12.79(26)$ & 8.95-16.63 & $\curlyvee$ \\
\hline ALT/AST increased & $76(27)$ & & $24.15(32)$ & $16.90-31.40$ & $\curlyvee$ \\
\hline Fatigue & $6,908(28)$ & $4,835.6 ?-8,980.4 ?$ & $103.00(32)$ & $72.10-133.90$ & $\curlyvee$ \\
\hline Anemia & $13,679(29)$ & $9,575-17,783$ & $921.10(33)$ & $348.60-1,494.32$ & $\curlyvee$ \\
\hline Nausea, vomiting & $5,246(28)$ & $3,672.2-6,819.8 ?$ & $39.60(34)$ & $27.72-51.48$ & $\curlyvee$ \\
\hline Stomatitis & 10,073.67 (30) & $7,051.57-13,095.77$ & $42.20(32)$ & $29.54-54.86$ & $\curlyvee$ \\
\hline Neutropenia & $9,910(29)$ & $6,937-12,883$ & $411.93(32)$ & $288.35-535.51$ & $\curlyvee$ \\
\hline Utilities & & & & & $\curlyvee$ \\
\hline PFS & $0.86(35-37)$ & $0.602-1$ & $0.86(35-37)$ & $0.602-1$ & $\beta$ \\
\hline PD & $0.71(35-37)$ & $0.497-0.923$ & $0.71(35-37)$ & $0.497-0.923$ & $\beta$ \\
\hline Discount rate, \% & \multicolumn{4}{|c|}{$3(0-5)(17)$} & $\beta$ \\
\hline
\end{tabular}

from 0 for death to 1 for perfect health. The utility values of this model were obtained from published literature with a health state similar to the HER2CLIMB trial (41-43).

\section{Sensitivity Analysis}

A series of deterministic sensitivity analyses were conducted to explore the impact of uncertainty in our assumptions on treatment efficacy, utilities, and cost. Variables in a deterministic sensitivity analysis were varied in the range confidence interval or $\pm 30 \%$. Besides, we investigated the possibility of TXT being cost-effective when the cost of tucatinib, utility value, and WTP threshold changed over a broader range. Based on the distribution characteristics of each parameter, gamma distributions were used for cost parameters, and the beta distributions were adopted for probability and health utility values. Then, we performed 1,000 Monte Carlo simulations to conduct probabilistic sensitivity analyses. The results of univariate sensitivity analyses were given as tornado diagram, and probabilistic sensitivity analyses were expressed as cost-effectiveness acceptability curves. We also analyzed the possibility of adding tucatinib being cost-effective in the brain metastasis subgroup.

\section{RESULTS}

\section{Base Case Results}

Table 2 shows the results of the basic analysis. The model projected that the patients treated with TXT yielded 1.10 QALYs, which was 0.21 QALYs more than patients receiving TX. The use of tucatinib, capecitabine plus trastuzumab cost an additional $\$ 19,022.97$, resulting in an ICER of $\$ 90,585.57$ per QALY compared with capecitabine plus trastuzumab for patients with MBC in China. In the United States, the ICER was $\$ 699,976.43$ per QALY.

\section{Subgroup Analysis}

For patients with brain metastasis, TXT costs $\$ 18,049.06$ more than TX with an additional 0.32 QALYs, resulting in an ICER of $\$ 56,403.31$ per QALY in China. From the US perspective, the ICER was $\$ 404,467.41$ per QALY.

\section{Sensitivity Analysis}

Figure 3 shows a tornado diagrams from a one-way sensitivity analysis. The most sensitive parameter is the price change of tucatinib in China and the United States, resulting in ICERs ranging from $\$ 77,189.02$ to $\$ 105,715.57$ and $\$ 527,420.51$ to $\$ 885,926.80$ per QALY, which are also higher than the WTP threshold set in the model. When perfect utilities were assigned to both stable and progressing disease states, the cost of TXT decreased to $\$ 87,708.57$ per QALY gained and $\$ 639,108.91$ per QALY, respectively.

The results of the probabilistic sensitivity analysis show that, in the United States, when the WTP threshold adjusted to $\$ 500,000$ or $\$ 800,000$, the probability that TXT is cost-effective compared with TX is 0.1 and $80.3 \%$, respectively (Table 2). For China, when the WTP threshold adjusted to $\$ 80,000$ or $\$ 100,000$, the probability of cost-effective in TXT group is 21.9 and $74.5 \%$, respectively (Table 2). In the United States, when the WTP threshold is $\$ 150,000 / \mathrm{QALY}$, and the unit price of tucatinib is 50 and $10 \%$ of the current price, the probability of cost-effective in the TXT group is 0 and $17.4 \%$, respectively (Table 2, Figure 4). With a WTP threshold of $\$ 30,447.09$ in China, the probability 
TABLE 2 | Summary of one- and multi-way deterministic and probabilistic sensitivity analyses.

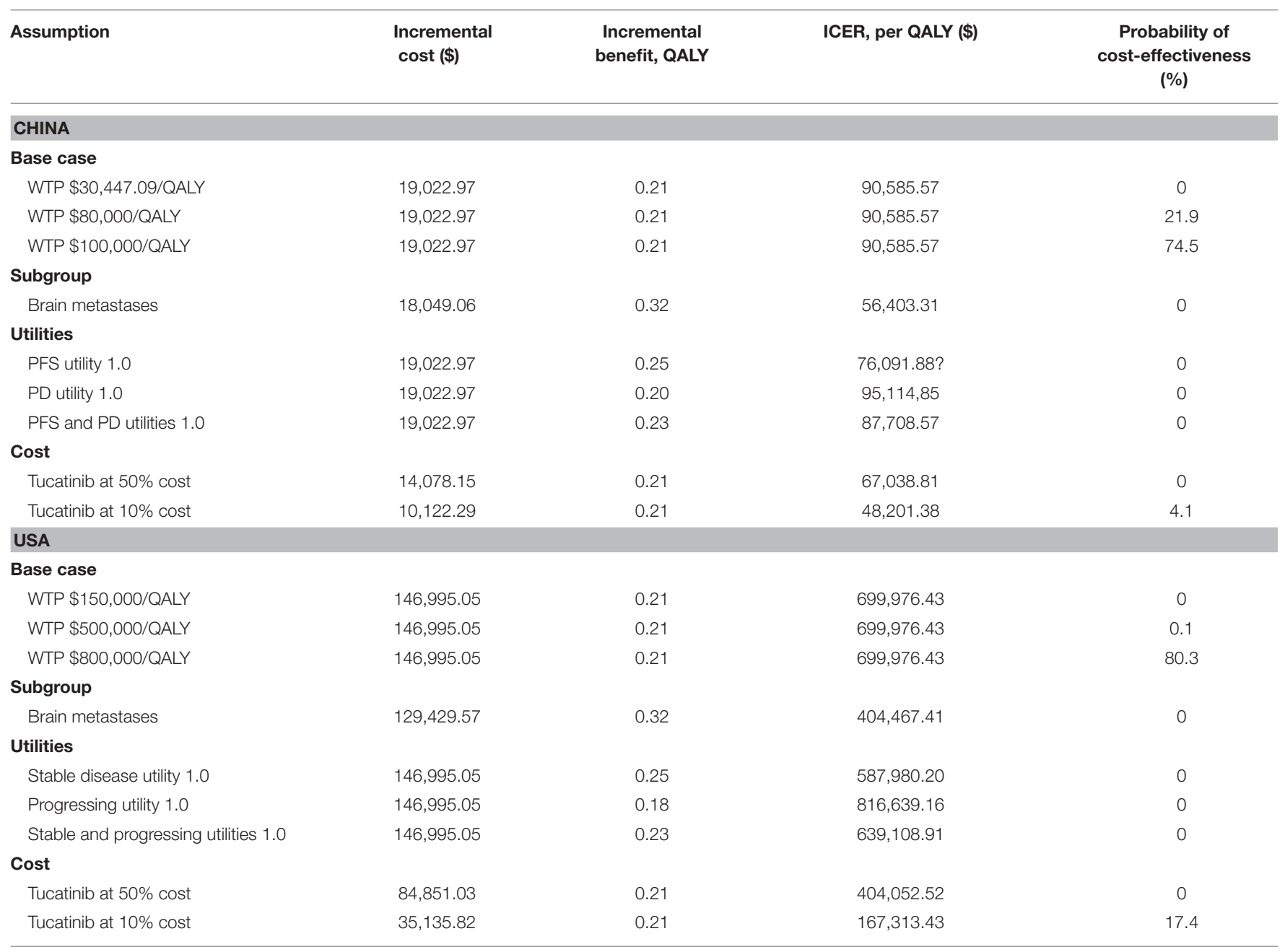

ICER, incremental cost-effectiveness ratio; OS, overall survival; PFS, progression-free survival; PD, progression disease; QALY, quality-adjusted life-years; WTP, willingness to pay.

of a cost-effective in the TXT group is $0 \%$. With the same discount ratio of tucatinib cost in China, the probabilities of costeffective in the TXT group were 0 and $4.1 \%$, respectively (Table 2, Figure 4).

\section{DISCUSSION}

To our knowledge, our study is the first cost effectiveness analysis of tucatinib, capecitabine plus trastuzumab compared with capecitabine plus trastuzumab for patients with Her-2 positive MBC from the United States and China. The ICER at base case estimate for TXT vs. TX was $\$ 699,976.43 / \mathrm{QALY}$ in the United States and \$90,585.57/QALY in China, both of which were higher than the WTP threshold of \$150,000/QALY and $\$ 30,447.09 / \mathrm{QALY}$, respectively. This indicates that TXT is unlikely to be a cost-effective treatment for MBC. The current incidence of $\mathrm{MBC}$ in the United States is 7.2 per 100,000 population at risk (44), and $20-25 \%$ of patients overexpress HER2 (8); therefore, if TXT therapy were universally implemented at current prices, it would add $\$ 699$ million per year to health care costs. For China, we estimated that the additional tucatinib treatment would increase the annual health burden by at least $\$ 724$ million because of the large population base $(8,45,46)$.

For patients with brain metastases, the increased cost of obtaining a QALY in the United States and China decreased to $\$ 395,373.81$ and $\$ 56,403.31$, respectively, which is mainly due to the more obvious difference of QALY in the brain metastasis group, suggesting the addition of tucatinib to capecitabine, and trastuzumab was most cost-effective for this subset. The survival benefit with tucatinib was observed in all subgroups tested of HER2CLIMB trial (15). Due to the lack of relevant data, we did not analyze subgroups other than brain metastases in our analysis. Therefore, from a more foresighted perspective, screening more appropriate patients will allow tucatinib treatment more likely to be cost-effective.

The univariable sensitivity showed that the parameter with the greatest influence on the ICER is the cost of tucatinib both in the United States and in China, which is consistent with the cost-effectiveness analysis of many innovative drugs in the 
A

Cost___Tucatinib
Cost___Trastuzumab
Cost_P_Trastuzumab
cost___PD_cycle
cost_P_PD_cycle
Cost_T_AEs
Cost_T_Capecitabine
Cost_P_Capecitabine
Cost_P_AEs
Cost_P_Hospital
Cost_T_Hospital
Utility_PD
Utility_PFS

B

Tornado Analysis(ICER)

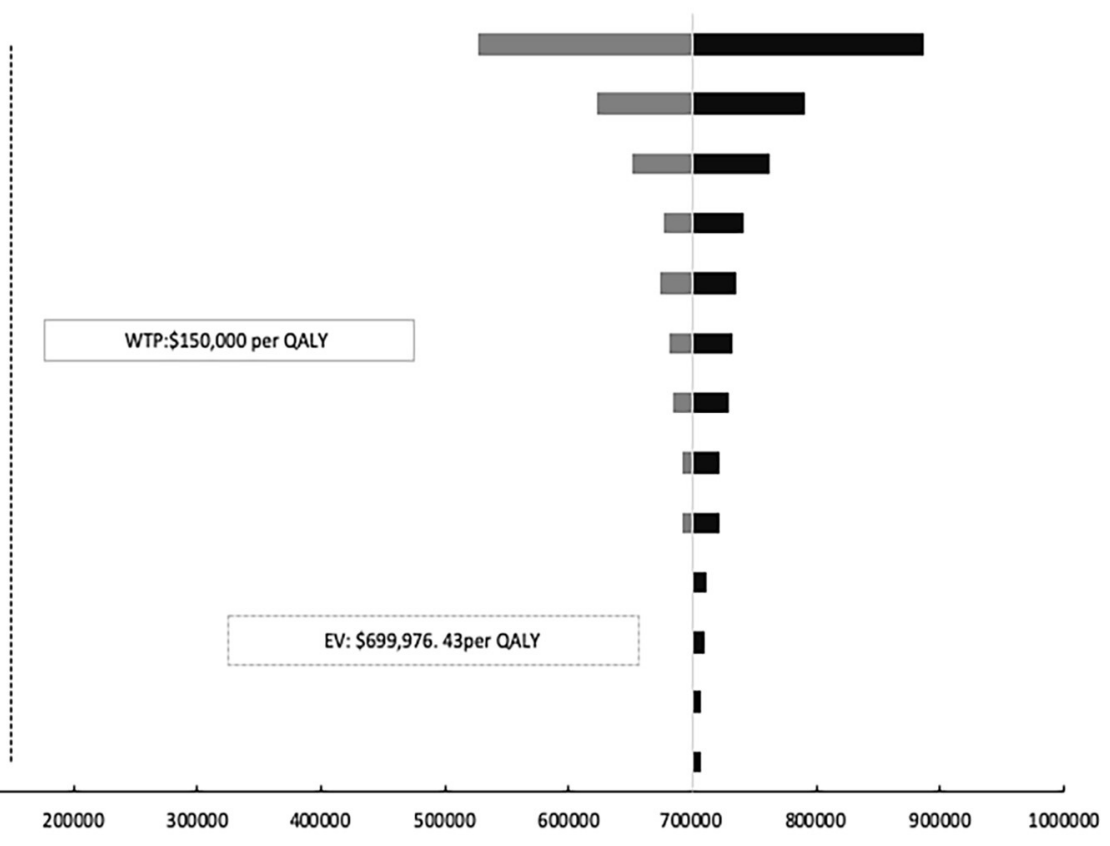

Tornado Analysis(ICER)

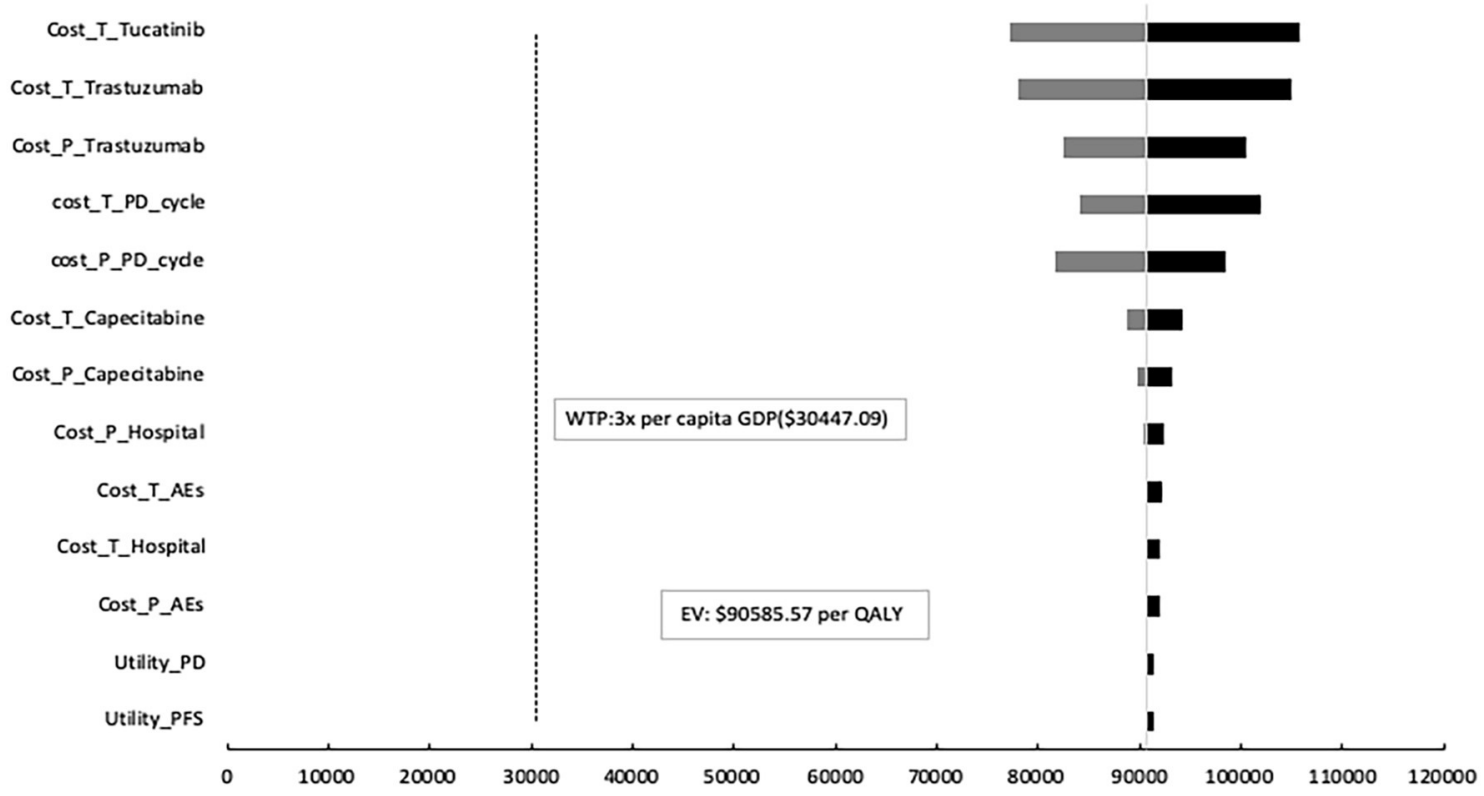

FIGURE 3 | One-way sensitivity analysis. This diagram shows the incremental cost effectiveness ratio (ICER) of TXT vs. TX for different model input parameters in the United States (A) and China (B), respectively. TXT, tucatinib, trastuzumab, and capecitabine; TX, trastuzumab plus capecitabine; PFS, progression-free survival; PD, progression disease; AEs, adverse events.

treatment of advanced tumors (47-49). Probabilistic sensitivity analysis shows that if the price of tucatinib decreased by $90 \%$, and the WTP threshold of China increases to $\$ 48,500$, the probability of cost-effectiveness of adding tucatinib would be increased to 50\%. Higher WTP may be achieved in some wealthy regions of China. According to statistics in 2018, there were four provincial-level administrative units in China (Beijing, Tianjin, Shanghai, and Jiangsu), whose three times GDP per capita were more than $\$ 48,500$, involving about 140 million people (50). The hypothetical price reduction strategy may apply to these regions. 


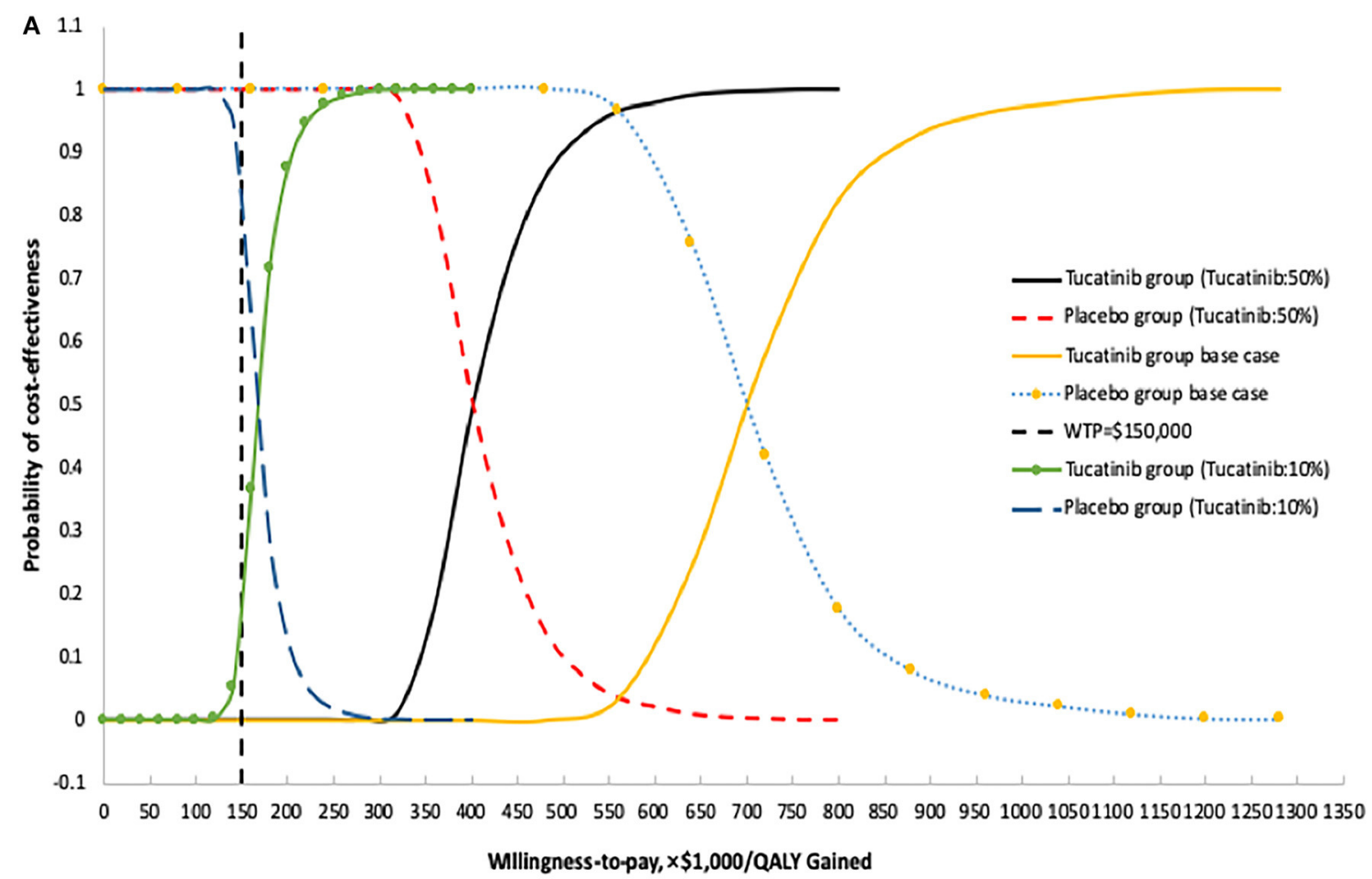

B

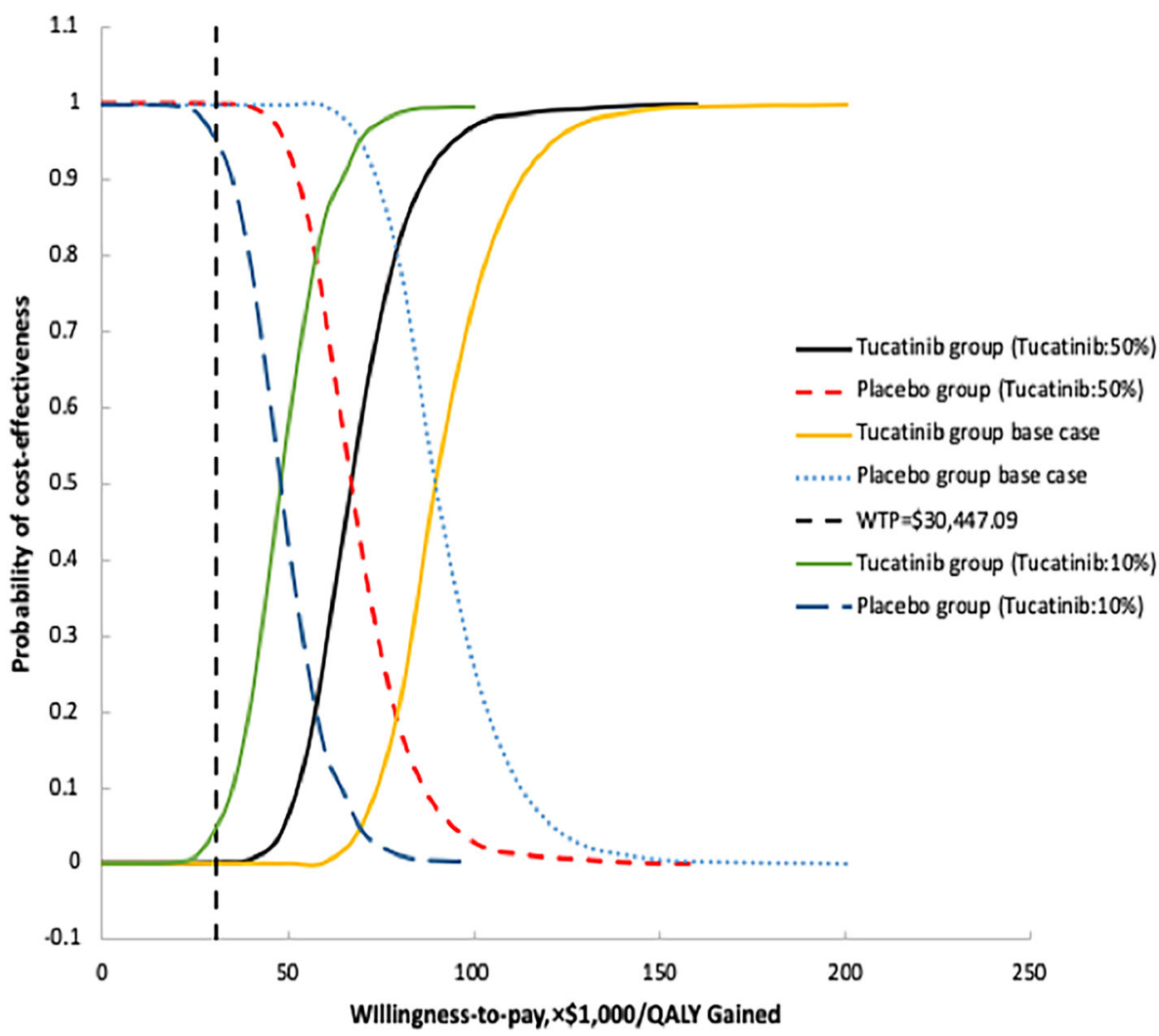

FIGURE 4 | Probabilistic sensitivity analysis for cost effectiveness of treatment strategies for HER2 positive metastatic breast cancer. Cost-effectiveness acceptable curves are showing the cost-effective probability of tucatinib, trastuzumab, and capecitabine at different prices from the United States (A) and Chinese (B) perspectives. The dotted vertical lines represent the willingness to pay thresholds. 
In January 2017, the State Council of China issued the National 13th 5 Year Plan to deepen the reform plan for the medical and health system, and many anti-cancer drugs price reduced after negotiations and were covered by medical insurance after entering the Chinese market. The medical insurance payment ratio of most anti-cancer drugs can reach about $70 \%$. For example, the price of Pertuzumab after approval by the China Food and Drug Administration is 1,342.86 yuan per milliliter, but after negotiated price reduction and medical insurance reimbursement, patients only need to pay about 107.14 yuan per milliliter, and the cost has dropped by more than $90 \%$. Changes in the actual cost of drugs provide tucatinib with the possibility to be cost-effective after entering China.

Tucatinib is not cost-effective for the United States at the current price, with the WTP threshold of \$150,000/QALY. When the cost is reduced to $\$ 7.03 / 100 \mathrm{mg}$, the ICER of TXT compared to TX will be equal to the current WTP. Besides, the costeffectiveness conclusion is the same between the two countries but with very different ICERs. Health care costs in China are far lower than in the United States, resulting in the ICER in the United States nearly eight times higher than in China. The limited transparency and absent federal control of drugs in American result in the highest drug costs worldwide (51). On May 11, 2018, the US administration released American Patients First to cut drug prices and decrease out-of-pocket payments (52). Significant price reduction or financial assistance is essential for patients to access innovative treatments and minimizing financial toxicity.

In fact, for patients with advanced cancer whose survival is limited, not only the TKIs, many anti-cancer drugs are not cost-effective due to their modest incremental benefit and high cost. Durkee et al. (53) published a cost of $\$ 472,668$ per QALY for patients treated with first-line Pertuzumab for patients with MBC. Another study by Liao et al. (43) reported a cost of $\$ 300,564$ per QALY for patients with fulvestrant plus anastrozole for hormone-receptor-positive MBC in postmenopausal women. Diaby et al. (54) reported a cost of $\$ 158,961.4$ per QALY for patients treated with second-line for $\mathrm{MBC}$. The use of innovative drugs that have been proven to be effective in clinical trials may lead to a substantial increase in medical expenditure, while the abandonment of these drugs means rejection of possible beneficial treatment (29). Therefore, cost-effectiveness analysis from different perspectives has become an important part of a broader discussion in how we allocate resources to treat cancer.

As in other cost-effectiveness analyses, our study has several limitations that are worth discussing. Firstly, the model input data come mainly from the results of the HER2CLIMB trial. For example, the Asian population accounts for only $4.4 \%$ of the HER2CLIMB trial (15), which may not accurately reflect the

\section{REFERENCES}

1. Siegel RL, Miller KD, Jemal A. Cancer statistics, 2020. ACS J. (2020) 70:7-30. doi: $10.3322 /$ caac. 21590 treatment effect of Chinese patients. Moreover, we did not have access to individual patients ' data, but survival data in our fitting curve were not significantly different from the results of the HER2CLIMB trial (Figure 2). Secondly, since the HER2CLIMB trial did not publish quality of life utility data, we assumed that the patients' quality of life was similar to that of previous studies (41-43). Also, we assumed the utility values of Chinese patients is equal to that of West. However, a range of $\pm 30 \%$ of utility values in the sensitivity analysis was used to analyze the effect of changes on the results. Thirdly, since tucatinib has not been marketed in China, the model's drug price is according to other drugs. We calculated the 50 and $90 \%$ off the model price of tucatinib, which will most likely include the lowest price value of tucatinib after approval. Fourth, we assumed in the model that all patients received the best supportive care after progression. This assumption is not completely consistent with clinical practice. However, patients in the HER2CLIMB trial have previously received trastuzumab, pertuzumab, and T-DM1 treatment, and $48 \%$ of these patients have brain metastases. The Nation Comprehensive Cancer Network guidelines and Chinese guidelines suggest the best supportive treatment as an option that may be taken into consideration for subsequent treatment $(55,56)$. Besides, the sensitivity analysis results show that the impact of subsequent treatment costs is considered limited.

In conclusion, from the payer's perspective in the United States and China, tucatinib is unlikely to be a costeffective treatment for $\mathrm{MBC}$ at the current price. New pricing, screening the dominant patients, generic drugs, and new payment systems are needed to support cost-effective treatment measures, and our analysis provides valuable recommendations.

\section{DATA AVAILABILITY STATEMENT}

All datasets generated for this study are included in the article/supplementary material.

\section{AUTHOR CONTRIBUTIONS}

QW coordinated and performed data analyses, reported study results, and drafted the manuscript. WL and MZ assisted with the analyses. $\mathrm{JH}$ and $\mathrm{PZ}$ contributed to the interpretation of the results and reviewed and revised the manuscript. All authors read and approved the final manuscript.

\section{FUNDING}

National Natural Science Foundation of China (No. 81572988) and Science \& Technology Department of Sichuan Province Funding Project (Nos. 2016FZ0108, 2018SZ0117). The 1.3.5 project for disciplines of excellence, West China Hospital, Sichuan University (No. ZYJC18010).

2. Chen W, Zheng R, Baade PD, Zhang S, Zeng H, Bray F, et al. Cancer statistics in China, 2015. CA Cancer J Clin. (2016) 66:115-32. doi: 10.3322/caac.21338

3. Bray F, Ferlay J, Soerjomataram I, Siegel RL, Torre LA, Jemal A. Global cancer statistics 2018: GLOBOCAN estimates of incidence and mortality worldwide 
for 36 cancers in 185 countries. CA Cancer J Clin. (2018) 68:394-424. doi: $10.3322 /$ caac. 21492

4. Wan X, Zhang Y, Tan C, Zeng X, Peng L. First-line nivolumab plus ipilimumab vs sunitinib for metastatic renal cell carcinoma: a costeffectiveness analysis. JAMA Oncol. (2019) 5:491-6. doi: 10.1001/jamaoncol. 2018.7086

5. Li J, Zhang BN, Fan JH, Pang Y, Zhang P, Wang SL, et al. A NationWide multicenter 10-year (1999-2008) retrospective clinical epidemiological study of female breast cancer in china. BMC Cancer. (2011) 11:364. doi: 10.1186/1471-2407-11-364

6. Cameron D, Piccart-Gebhart MJ, Gelber RD, Procter M, Goldhirsch A, de Azambuja E, et al. 11 years' follow-up of trastuzumab after adjuvant chemotherapy in HER2-positive early breast cancer: final analysis of the HERceptin Adjuvant (HERA) trial. Lancet. (2017) 389:1195-205. doi: 10.1016/S0140-6736(16)32616-2

7. Perez EA, Romond EH, Suman VJ, Jeong JH, Sledge G, Geyer CE, Jr., et al. Trastuzumab plus adjuvant chemotherapy for human epidermal growth factor receptor 2-positive breast cancer: planned joint analysis of overall survival from NSABP B-31 and NCCTG N9831. J Clin Oncol. (2014) 32:3744-52. doi: 10.1200/JCO.2014.55.5730

8. Owens MA, Horten BC, Da Silva MM. HER2 amplification ratios by fluorescence in situ hybridization and correlation with immunohistochemistry in a cohort of 6556 breast cancer tissues. Clin Breast Cancer. (2004) 5:63-9. doi: 10.3816/CBC.2004.n.011

9. Cronin KA, Harlan LC, Dodd KW, Abrams JS, Ballard-Barbash R. Population-based estimate of the prevalence of HER-2 positive breast cancer tumors for early stage patients in the US. Cancer Invest. (2010) 28:963-8. doi: 10.3109/07357907.2010.496759

10. Piccart-Gebhart MJ, Procter M, Leyland-Jones B, Goldhirsch A, Untch M, Smith I, et al. Trastuzumab after adjuvant chemotherapy in HER2-positive breast cancer. N Engl J Med. (2005) 353:1659-72. doi: 10.1056/NEJMoa0 52306

11. Loibl S, Gianni L. HER2-positive breast cancer. Lancet. (2017) 389:2415-29. doi: 10.1016/S0140-6736(16)32417-5

12. Duchnowska R, Loibl S, Jassem J. Tyrosine kinase inhibitors for brain metastases in HER2-positive breast cancer. Cancer Treat Rev. (2018) 67:71-7. doi: 10.1016/j.ctrv.2018.05.004

13. Borges VF, Ferrario C, Aucoin N, Falkson C, Khan Q, Krop I, et al. Tucatinib combined with ado-trastuzumab emtansine in advanced ERBB2/HER2positive metastatic breast cancer: a phase $1 \mathrm{~b}$ clinical trial. JAMA Oncol. (2018) 4:1214-20. doi: 10.1001/jamaoncol.2018.1812

14. U.S.Food and Drug Administration (2017). Available online at: https://www. accessdata.fda.gov/scripts/opdlisting/oopd/detailedIndex.cfm?cfgridkey= 570716 (accessed March 30, 2020).

15. Murthy RK, Loi S, Okines A, Paplomata E, Hamilton E, Hurvitz SA, et al. Tucatinib, Trastuzumab, and Capecitabine for HER2positive metastatic breast cancer. N Engl J Med. (2020) 382:597-609. doi: 10.1056/NEJMoa1914609

16. FDA Approves First New Drug Under International Collaboration, A Treatment Option for Patients with HER2-Positive Metastatic Breast Cancer (2020). Available online at: https://www.fda.gov/news-events/press-announcements/ fda-approves-first-new-drug-under-international-collaboration-treatmentoption-patients-her2 (accessed April 18, 2020).

17. Salgia R, Mambetsariev I, Hewelt B, Achuthan S, Li H, Poroyko $\mathrm{V}$, et al. Modeling small cell lung cancer (SCLC) biology through deterministic and stochastic mathematical models. Oncotarget. (2018) 9:26226-42. doi: 10.18632/oncotarget.25360

18. Neumann PJ, Cohen JT, Weinstein MC. Updating cost-effectiveness-the curious resilience of the \$50,000-per-QALY threshold. N Engl J Med. (2014) 371:796-7. doi: 10.1056/NEJMp1405158

19. Wu Q, Liao W, Huang J, Zhang P, Zhang N, Li Q. Cost-effectiveness analysis of gemcitabine plus cisplatin versus docetaxel, cisplatin and fluorouracil for induction chemotherapy of locoregionally advanced nasopharyngeal carcinoma. Oral Oncol. (2020) 103:104588. doi: 10.1016/j.oraloncology.2020.104588

20. Half-Cycle Correction. York; York Health Economics Consortium. (2016). Available onlien at: http://www.yhec.co.uk/glossary/half-cycle-correction/ (accessed April 20, 2020).
21. Hoyle MW, Henley W. Improved curve fits to summary survival data: application to economic evaluation of health technologies. BMC Med Res Methodol. (2011) 11:139. doi: 10.1186/1471-2288-11-139

22. Diaby V, Adunlin G, Montero AJ. Survival modeling for the estimation of transition probabilities in model-based economic evaluations in the absence of individual patient data: a tutorial. Pharmacoeconomics. (2014) 32:101-8. doi: 10.1007/s40273-013-0123-9

23. Basu S, Yudkin JS, Sussman JB, Millett C, Hayward RA. Alternative strategies to achieve cardiovascular mortality goals in china and india: a microsimulation of target- versus risk-based blood pressure treatment. Circulation. (2016) 133:840-8. doi: 10.1161/CIRCULATIONAHA.115.019985

24. Arias E, Heron M, Xu J. United States life tables, 2014. Natl Vital Stat Rep. (2017) 66:1-64.

25. IBM. RED BOOK Online $\mathbb{R} /$ Micromedex. (2020). Available online at: http:// www.micromedexsolutions.com (accessed April 20, 2020).

26. Tamura K, Aiba K, Saeki T, Nakanishi Y, Kamura T, Baba H, et al. Breakthrough chemotherapy-induced nausea and vomiting: report of a nationwide survey by the CINV Study Group of Japan. Int J Clin Oncol. (2017) 22:405-12. doi: 10.1007/s10147-016-1069-7

27. Sorensen SV, Goh JW, Pan F, Chen C, Yardley D, Martín M, et al. Incidence-based cost-of-illness model for metastatic breast cancer in the United States. Int J Technol Assess Health Care. (2012) 28:12-21. doi: 10.1017/S026646231100064X

28. Cadier B, Bulsei J, Nahon P, Seror O, Laurent A, Rosa I, et al. Early detection and curative treatment of hepatocellular carcinoma: a cost-effectiveness analysis in France and in the United States. Hepatology. (2017) 65:1237-48. doi: 10.1002/hep. 28961

29. Liao W, Huang J, Hutton D, Zhu G, Wu Q, Wen F, et al. Cost-effectiveness analysis of cabozantinib as second-line therapy in advanced hepatocellular carcinoma. Liver Int. (2019) 39:2408-16. doi: 10.1111/liv.14257

30. Xie J, Diener M, Sorg R, Wu EQ, Namjoshi M. Cost-effectiveness of denosumab compared with zoledronic acid in patients with breast cancer and bone metastases. Clin Breast Cancer. (2012) 12:247-58. doi: 10.1016/j.clbc.2012.04.001

31. Mistry R, May JR, Suri G, Young K, Brixner D, Oderda G, et al. CostEffectiveness of ribociclib plus letrozole versus palbociclib plus letrozole and letrozole monotherapy in the first-line treatment of postmenopausal women with HR+/HER2- advanced or metastatic breast cancer: a U.S. payer perspective. J Manag Care Spec Pharm. (2018) 24:514-23. doi: 10.18553/jmcp.2018.24.6.514

32. Rashid N, Koh HA, Baca HC, Lin KJ, Malecha SE, Masaquel A. Economic burden related to chemotherapy-related adverse events in patients with metastatic breast cancer in an integrated health care system. Breast Cancer. (2016) 8:173-81. doi: 10.2147/BCTT.S105618

33. Perrin A, Sherman S, Pal S, Chua A, Gorritz M, Liu Z, et al. Lifetime cost of everolimus vs axitinib in patients with advanced renal cell carcinoma who failed prior sunitinib therapy in the US. J Med Econ. (2015) 18:200-9. doi: 10.3111/13696998.2014.985789

34. Ding H, Fang L, Xin W, Tong Y, Zhou Q, Huang P. Cost-effectiveness analysis of fulvestrant versus anastrozole as first-line treatment for hormone receptorpositive advanced breast cancer. Eur J Cancer Care. (2017) 26:e12733. doi: $10.1111 /$ ecc. 12733

35. Shi G, Park SH, Ren H, Xue M, Lu X, Dong P, et al. Cost analysis for different sequential treatment regimens for metastatic renal cell carcinoma in China. $J$ Med Econ. (2018) 21:1150-8. doi: 10.1080/13696998.2018.1515769

36. Gu X, Zhang Q, Chu YB, Zhao YY, Zhang YJ, Kuo D, et al. Cost-effectiveness of afatinib, gefitinib, erlotinib and pemetrexed-based chemotherapy as first-line treatments for advanced non-small cell lung cancer in China. Lung Cancer. (2019) 127:84-9. doi: 10.1016/j.lungcan.2018.11.029

37. Chongqing T, Liubao P, Xiaohui Z, Jianhe L, Xiaomin W, Gannong C, et al. Cost-utility analysis of the newly recommended adjuvant chemotherapy for resectable gastric cancer patients in the 2011 Chinese National Comprehensive Cancer Network (NCCN) clinical practice guidelines in oncology: Gastric Cancer. (2014) 32:235-43. doi: 10.1007/s40273-013-0065-2

38. US Department of Labor. Calculators. (2020). Available online at: http://www. bls.gov/data/\#calculators (accessed June 10, 2020).

39. National Bureau of Statistics of China. Health Care and Personal Articles of Consumer Price Indices (2020). Available online at: 
http://data.stats.gov.cn/english/easyquery.htm? $\mathrm{cn}=\mathrm{C} 01 \quad$ (accessed June 10, 2020).

40. Drummond MF, Sculpher MJ, Claxton K, Stoddart GL, Torrance GW. Methods for the Economic Evaluation Of Health Care Programmes. Oxford, UK: Oxford University Press (2015).

41. Lloyd A, Nafees B, Narewska J, Dewilde S, Watkins J. Health state utilities for metastatic breast cancer. Br J Cancer. (2006) 95:683-90. doi: $10.1038 /$ sj.bjc. 6603326

42. Shiroiwa T, Fukuda T, Shimozuma K, Mouri M, Hagiwara Y, Doihara H, et al. Long-term health status as measured by EQ-5D among patients with metastatic breast cancer: comparison of first-line oral S-1 and taxane therapies in the randomized phase III SELECT BC trial. Qual Life Res. (2017) 26:445-53. doi: 10.1007/s11136-016-1388-1

43. Liao W, Huang J, Wu Q, Wen F, Zhang N, Zhou K, et al. First-line fulvestrant plus anastrozole for hormone-receptor-positive metastatic breast cancer in postmenopausal women: a cost-effectiveness analysis. Breast Cancer. (2020) 27:399-404. doi: 10.1007/s12282-019-01034-6

44. National Cancer Institute. Surveillance, Epidemiology, and end Results Program. SEER Explorer (2019). Available online at: https://seer.cancer.gov/ explorer/application.php (accessed February 10, 2020).

45. Zheng RS, Sun KX, Zhang SW, Zeng HM, Zou XN, Chen R, et al. [Report of cancer epidemiology in China, 2015]. Zhonghua Zhong Liu Za Zhi. (2019) 41:19-28. doi: 10.3760/cma.j.issn.0253-3766.2019.01.005

46. Gonzalez-Angulo AM, Morales-Vasquez F, Hortobagyi GN. Overview of resistance to systemic therapy in patients with breast cancer. Adv Exp Med Biol. (2007) 608:1-22. doi: 10.1007/978-0-387-74039-3_1

47. Goldstein DA, Ahmad BB, Chen Q, Ayer T, Howard DH, Lipscomb J, et al. Cost-effectiveness analysis of regorafenib for metastatic colorectal cancer. $J$ Clin Oncol. (2015) 33:3727-32. doi: 10.1200/jco.2015.33.15_suppl.6613

48. Wan XM, Peng LB, Ma JA, Li YJ. Economic evaluation of nivolumab as a second-line treatment for advanced renal cell carcinoma from US and Chinese perspectives. Cancer. (2017) 123:2634-41. doi: 10.1002/cncr.30666

49. Liao M, Jiang Q, Hu H, Han J, She L, Yao L, et al. Cost-effectiveness analysis of utidelone plus capecitabine for metastatic breast cancer in China. J Med Econ. (2019) 22:584-92. doi: 10.1080/13696998.2019.1588125
50. National bureau of statistics of China (2019). Available online at: http://data. stats.gov.cn/index.htm (accessed February 4, 2020).

51. Prasad V, De Jesús K, Mailankody S. The high price of anticancer drugs: origins, implications, barriers, solutions. Nat Rev Clin Oncol. (2017) 14:38190. doi: $10.1038 /$ nrclinonc. 2017.31

52. Burki TK. A new strategy to reduce US drug prices. Lancet Oncol. (2018). doi: 10.1016/S1470-2045(18)30374-7. [Epub ahead of print].

53. Durkee BY, Qian Y, Pollom EL, King MT, Dudley SA, Shaffer JL, et al. Cost-effectiveness of pertuzumab in human epidermal growth factor receptor 2-positive metastatic breast cancer. J Clin Oncol. (2016) 34:902-9. doi: 10.1200/JCO.2015.62.9105

54. Diaby V, Alqhtani H, van Boemmel-Wegmann S, Wang CY, Ali AA, Balkrishnan R, et al. A cost-effectiveness analysis of trastuzumabcontaining treatment sequences for HER-2 positive metastatic breast cancer patients in Taiwan. Breast. (2020) 49:141-8. doi: 10.1016/j.breast.2019. 11.012

55. NCCN clinical practice guidelines in Oncology. Breast Cancer(2020.V4) (2020). Available online at: https://www.nccn.org/ professionals/physician_gls/pdf/breast.pdf (accessed June 11, 2020).

56. Guidelines of Chinese Society of Clinical Oncology(CSCO). Breast Cancer 2019 (2019). Available online at: http://meeting.csco.org.cn/pdf/web/viewer. html?file=/upload/Periodical/201910/201910814559.pdf (accessed June 10, 2020).

Conflict of Interest: The authors declare that the research was conducted in the absence of any commercial or financial relationships that could be construed as a potential conflict of interest.

Copyright (c) $2020 \mathrm{Wu}$, Liao, Zhang, Huang, Zhang and Li. This is an open-access article distributed under the terms of the Creative Commons Attribution License (CC $B Y)$. The use, distribution or reproduction in other forums is permitted, provided the original author(s) and the copyright owner(s) are credited and that the original publication in this journal is cited, in accordance with accepted academic practice. No use, distribution or reproduction is permitted which does not comply with these terms. 